\title{
Uma Análise de Problemas de Função Afim Fundamentada na Teoria dos Campos Conceituais
}

\author{
An Analysis of Affine Function Problems Based on Conceptual Fields Theory
}

\author{
Clarice de Almeida Miranda ${ }^{\text {ab }}$, Veridiana Rezende ${ }^{\text {bc }}$; Clélia Maria Ignatius Nogueira ${ }^{\text {ac }}$ \\ aUniversidade Estadual do Oeste do Paraná, Programa de Pós-Graduação Stricto Sensu em Educação em Ciências e Educação Matemática. PR, Brasil. \\ bUniversidade Estadual do Paraná, Colegiado de Matemática. PR, Brasil. \\ ‘Universidade Estadual Paraná, Programa de Pós-Graduação Stricto Sensu em Educação Matemática. PR, Brasil. \\ *E-mail: voclelia@gmail.com.
}

\begin{abstract}
Resumo
Apresenta-se neste artigo os resultados de uma pesquisa que teve por objetivo analisar estruturas de problemas de função afim e estabelecer sua classificação. Para o seu desenvolvimento, foram considerados os problemas resolvidos no capítulo específico sobre função afim de um livro didático de Matemática do $1^{\circ}$ ano do Ensino Médio. A análise dos dados foi realizada com base na teoria dos Campos Conceituais, com foco nas relações entre as medidas identificadas nos enunciados das situações-problema de função afim. Os resultados mostram que é possível analisar problemas de função afim como problemas mistos, envolvendo estruturas dos campos aditivo e multiplicativo ou problemas puramente multiplicativos. Dentre as situações-problema analisadas, 02 (duas) foram identificadas como estritamente do campo multiplicativo, da classe proporção simples; e 03 (três) são mistas, sendo 01 (uma) do tipo proporção simples e composição de medidas e 02 (duas) de proporção simples e transformação de medidas.
\end{abstract}

Palavras-chave: Didática da Matemática. Função Afim. Estruturas Aditivas. Estruturas Multiplicativas. Problemas Mistos.

\begin{abstract}
This article presents the results of a research that aimed at analyzing structures problems of affine function and establish their typology. For the investigation development, the problems solved in the specific chapter on affine function, present in a Mathematics textbook of the High School $1^{s t}$ grade. Data analysis was carried out based on the Conceptual Fields theory, looking at the types of relationships involved between the measures identified in the problem situations statements that involve affine function concept. Findings show that is possible to analyze similar affine function problems as mixed problems, involving structures of the Additive and Multiplicative fields or purely multiplicative problems. Among the problem situations analyzed, 03 (three) belong to the mixed type, one of them as simple proportion type and measures composition, and two of them as simple proportion type and measurement transformation.
\end{abstract}

Keywords: Didactics of Mathematics. Affine Function. Additive Structures. Multiplicative Structures. Mixed Problems.

\section{Introdução}

Tradicionalmente, o ensino de função privilegia o aspecto algébrico, e está focalizado em técnicas e algoritmos para a resolução das situações propostas (Campiteli \& Campiteli, 2006). Com base em Vergnaud (1990), entendemos que a aprendizagem de um conceito ocorre durante todo o processo escolar, a partir de diferentes situações vivenciadas pelos estudantes.

No que se refere às funções, a Base Nacional Comum Curricular - BNCC (Brasil, 2019), documento que norteia a Educação Básica brasileira, recomenda que as ideias associadas a esse conceito devam ser desenvolvidas com as crianças desde os Anos Iniciais - Ensino Fundamental. Tal desenvolvimento deve ocorrer por meio de situações relacionadas ao reconhecimento de padrões (regularidade), envolvendo elementos ausentes em sequências recursivas de números naturais, objetos ou figuras, entre outras (Brasil, 2019). Tais ideias devem ser aprofundadas no decorrer dos anos escolares para que, a partir de um processo contínuo de ensino e de diferentes situações associadas às ideias de função, esse conceito seja institucionalizado no $9^{\circ}$ ano do Ensino Fundamental e aprofundado no $1^{\circ}$ ano do Ensino Médio (Brasil, 2019).

$\mathrm{Na}$ teoria dos campos conceituais (Vergnaud, 1990), um dos conceitos centrais é o de campo conceitual, definido como um conjunto de situações, conceitos, propriedades, teoremas e representações simbólicas interligados a um mesmo conceito. Dentre os campos conceituais investigados por Vergnaud, dois deles recebem destaque (Vergnaud, 1990; 2009a; 2009b) - o das estruturas aditivas e o das estruturas multiplicativas, para os quais o pesquisador apresenta classes de situações bem definidas.

Vergnaud (1993) adota a ideia de situações como um conjunto de tarefas a serem realizadas pelo sujeito, e as situações mais complexas podem ser entendidas como um conjunto de subtarefas. No que se refere às relações entre as situações e a elaboração do conceito, o pesquisador considera dois aspectos principais: a variedade (diversidade de situações 
relacionadas ao conceito) e a história (a progressão de apresentação das situações). Nesta investigação, interessamonos principalmente pelo primeiro aspecto, associado à variedade de situações envolvidas em um Campo Conceitual e necessária para a compreensão do respectivo conceito.

Com estes pressupostos e considerando que o mapeamento do Campo Conceitual da função afim é o foco de investigação do grupo de pesquisa do qual participamos e lideramos Grupo de Estudos e Pesquisas em Didática da Matemática GEPeDiMa, para a pesquisa aqui relatada, buscamos alcançar o seguinte objetivo: analisar estruturas de problemas de função afim e estabelecer sua classificação. Para isso, levamos em consideração três aspectos: i) o conceito de função afim está diretamente relacionado às operações de adição e multiplicação, possível de visualizar principalmente a partir de sua representação algébrica ; ii) a classificação e a análise das estruturas dos problemas dos campos conceituais aditivo e multiplicativo, estabelecidas por Vergnaud (1990; 2009a; 2009 b); iii) os problemas mistos - concebidos por Vergnaud (2009b) como aqueles que envolvem as operações de adição/ subtração e multiplicação/divisão.

A investigação apresentada neste texto refere-se à análise das estruturas de problemas de função afim propostos em um livro didático de Matemática do $1^{\circ}$ ano do Ensino Médio, e faz parte dos primeiros resultados da investigação de Mestrado da primeira autora, sob a orientação das demais autoras.

\section{Alguns Aspectos Sobre a Teoria dos Campos Conceituais}

A Teoria dos Campos Conceituais (TCC) tem por escopo estudar o processo de aquisição do conhecimento pelo sujeito, especialmente do estudante em fase escolar. Um dos pressupostos desta teoria é que as competências de um sujeito se desenvolvem ao longo do tempo, por meio de diferentes situações vivenciadas, ou seja, a partir de suas experiências (Vergnaud, 2009a).

Vergnaud (2009a) corrobora com a afirmação da teoria piagetiana de que conhecimento é adaptação, o sujeito se adapta às situações por meio de uma evolução da organização de sua atividade. Quando essa organização é invariante em uma classe de situações, ela é denominada pelo pesquisador de esquema, conceito também adotado da Psicologia Genética. Em outras palavras, segundo a TCC, o conhecimento ocorre a partir da adaptação dos esquemas mobilizados pelo sujeito face a uma dada classe de situações.

Por conseguinte, aprender apresenta-se como o movimento de adaptação às situações e de desenvolvimento de competências e novos esquemas, necessários para conseguir obter êxito na realização das tarefas que constituem novas situações. Em contexto de aprendizagem sempre existirá um conjunto de situações para as quais o sujeito já possui um esquema organizado e competências necessárias para resolvêlas, e um conjunto de situações em que o sujeito não possui as competências necessárias para resolvê-las, levando à manifestação de diferentes esquemas, adequados ou não, para uma possível resolução (Vergnaud, 2009a).

Para Vergnaud (2009a), um conceito não se desenvolve sozinho e isoladamente. Nessa direção, o pesquisador estabelece que um determinado conceito se desenvolve mediante a terna (S, I, R), sendo: S um conjunto de situações que dão sentido ao conceito em questão; I um conjunto de invariantes (propriedades e relações) utilizado na resolução das situações e nos esquemas dos sujeitos; R um conjunto de representações simbólicas usado na representação do próprio conceito e suas relações, bem como para as situações e esquemas envolvidos.

Ao longo de suas pesquisas, dois Campos Conceituais recebem destaque por serem bem estabelecidos por Vergnaud, sendo um deles associado à operação de adição e o outro à operação de multiplicação, os quais denominou de Campo Conceitual das Estruturas Aditivas e Campo Conceitual das Estruturas Multiplicativas, respectivamente. Cada um destes campos conceituais caracteriza classes de situações bem definidas que dão sentido aos conceitos de adição e multiplicação.

$\mathrm{Na}$ análise de problemas dos campos conceituais aditivo e multiplicativo, Vergnaud (2009b) realizou estudos das relações entre as medidas presentes em situações que envolvem relações binárias, relações ternárias e relações quaternárias, ou seja, que colocam em relação duas, três e quatro medidas, respectivamente. Essas relações são frequentemente representadas pelo pesquisador por um esquema relacional (Vergnaud, 2009b) que permite explicitar os tipos de relações entre medidas e deduções que podem ser consideradas para cada tipo de situação.

Esse esquema relacional permite representar as relações dos elementos de um problema utilizando códigos, conforme a Figura 1.

Figura 1 - Códigos utilizados em um esquema relacional

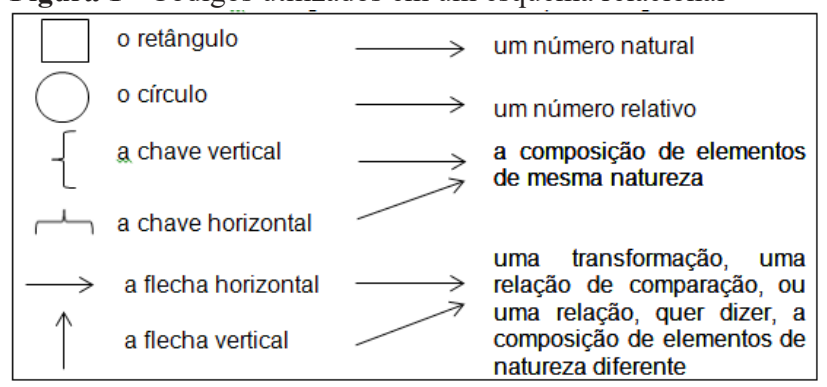

Fonte: Vergnaud (2009b, p. 201).

Esses códigos auxiliam a representar o cálculo relacional de uma determinada situação a ser analisada. As constatações, deduções ou inferências e construções realizadas para a compreensão das situações são denominadas por cálculo relacional (Vergnaud, 2009b). Segundo Vergnaud (2009b, p. 37), há dois aspectos importantes do cálculo relacional: "compor duas relações e tomar a recíproca de uma relação". Assim, “[...] o cálculo relacional é baseado não apenas nas 
propriedades das relações (simetria, transitividade etc.), mas também nas ligações que as relações têm entre si, isto é, sobre as relações entre relações" (Vergnaud, 2009b, p. 93).

A partir dos estudos de problemas que podem ser resolvidos com operações de adição ou subtração, ou uma combinação destas operações, Vergnaud (2009b, p.200) constatou que "as relações aditivas são relações ternárias que podem ser encadeadas de diversas maneiras e resultar em uma grande variedade de estruturas aditivas".

As classes de situações aditivas estabelecidas por
Vergnaud (1993) são: 1) Composição de duas medidas em uma terceira; 2) Transformação de uma medida inicial em uma medida final; 3) Relação de comparação entre duas medidas; 4) Composição de duas transformações; 5) Transformação de uma relação; 6) Composição de duas relações.

O Quadro 1 contém um exemplo de situação e o esquema relacional referente a cada uma das classes citadas, não tendo como objetivo, neste momento, analisar cada uma dessas classes de situações, mas de estabelecer uma referência à teoria abordada neste estudo.

Quadro 1 - Estrutura de problemas do campo aditivo

\begin{tabular}{|c|c|c|}
\hline Classe de Problemas & Esquema Relacional & Exemplo de Situação \\
\hline $\begin{array}{l}\text { Composição de } \\
\text { medidas }\end{array}$ & $\frac{a}{b}$ & $\begin{array}{l}\text { Hoje na minha sala de aula estão presentes } 3 \text { meninos e } 5 \text { meninas. Quantas } \\
\text { pessoas vieram na aula hoje? (Zanella \& Barros, 2014, p. 27). }\end{array}$ \\
\hline $\begin{array}{l}\text { Transformação de } \\
\text { medidas }\end{array}$ & $a$ & $\begin{array}{l}\text { Havia } 17 \text { pessoas dentro de um ônibus, subiram 4. Quantas pessoas estão ali } \\
\text { dentro, agora? (Vergnaud, 2009b, p. 207). }\end{array}$ \\
\hline $\begin{array}{l}\text { Relação entre } \\
\text { medidas }\end{array}$ & & $\begin{array}{l}\text { Paulo tem } 8 \text { bolinhas de gude. Tiago tem } 5 \text { a menos que Paulo. Quantas } \\
\text { bolinhas de gude tem Tiago? (Adaptado de Vergnaud (2009b, p. 203)). }\end{array}$ \\
\hline $\begin{array}{l}\text { Composição de } \\
\text { transformações }\end{array}$ & & $\begin{array}{l}\text { João jogou duas partidas de bolinha de gude. Na primeira partida ele ganhou } \\
16 \text { bolinhas. Na segunda partida, ganhou } 9 \text {. Ao final, o que aconteceu? } \\
\text { (Vergnaud, 2009b, p. 217). }\end{array}$ \\
\hline $\begin{array}{l}\text { Transformação de } \\
\text { relações }\end{array}$ & & $\begin{array}{l}\text { No campeonato brasileiro de Voleibol, a seleção do Rio tinha } 5 \text { pontos a } \\
\text { menos do que a seleção de Minas. Nessa última rodada, a diferença entre as } \\
\text { pontuações das equipes diminuiu } 3 \text { pontos. Quantos pontos a mais ou a menos } \\
\text { tem a seleção do Rio em relação à de Minas? (Zanella\& Barros, 2014, p. 48). }\end{array}$ \\
\hline $\begin{array}{l}\text { Composição de } \\
\text { relações }\end{array}$ & & $\begin{array}{l}\text { Denise tem a mais do que Marli. Por sua vez, Marli tem a mais que Lilian. } \\
\text { Quanto Denise tem a mais do que Lilian? (Zanella \& Barros, 2014, p. 55). }\end{array}$ \\
\hline
\end{tabular}

Fonte: Dados da pesquisa.

Cada classe de situação possui um grau de dificuldade próprio, levando em consideração o tipo de relação estabelecida entre os elementos da situação. Essa dificuldade em resolver a situação também pode ser diferenciada em problemas que pertencem a uma mesma classe, ao considerarmos as subclasses possíveis para cada classe.

Por exemplo, em problemas de transformação de medidas, que podem ser representados pelo esquema relacional a seguir (Figura 2), as subclasses de problemas relacionadas à classe de transformação são definidas "conforme seja a transformação b positiva ou negativa; conforme seja a pergunta concernente ao estado final c (conhecendo-se $\mathrm{a}$ e b), à transformação $b$ (conhecendo-se a e c), ao estado inicial (conhecendo-se b e c)" (Vergnaud, 2009b, p. 207).

Figura 2 - Esquema relacional de uma transformação de medidas

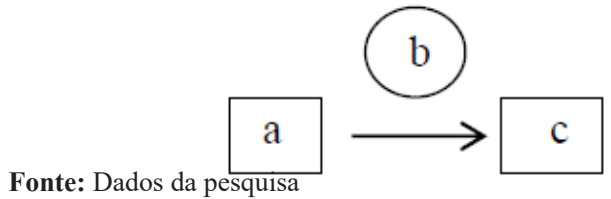


Além da medida desconhecida, outra variação do problema refere-se aos números relativos de cada classe de situação (exceto composição de medidas), que podem ser positivos ou negativos, modificando (ou não) o grau de dificuldade da situação. No quadro 2, as letras, e representam números relativos, e cada valor ou sinal que assumem pode ser um fator modificador e dificultar a compreensão da situação pelo estudante.
Para o campo conceitual multiplicativo, Vergnaud estabelece cinco classes de problemas: 1) Proporção simples ou Isomorfismo de medidas; 2) Comparação multiplicativa; 3) Produto de medidas ou produto cartesiano; 4) Proporção dupla ou Função bilinear; 5) Proporção múltipla (Vergnaud, 2009b, Gitirana, 2014).

O Quadro 2 contém o esquema relacional e um exemplo para as primeiras classes identificadas no campo multiplicativo.

Quadro 2 - Estruturas de problemas do campo multiplicativo

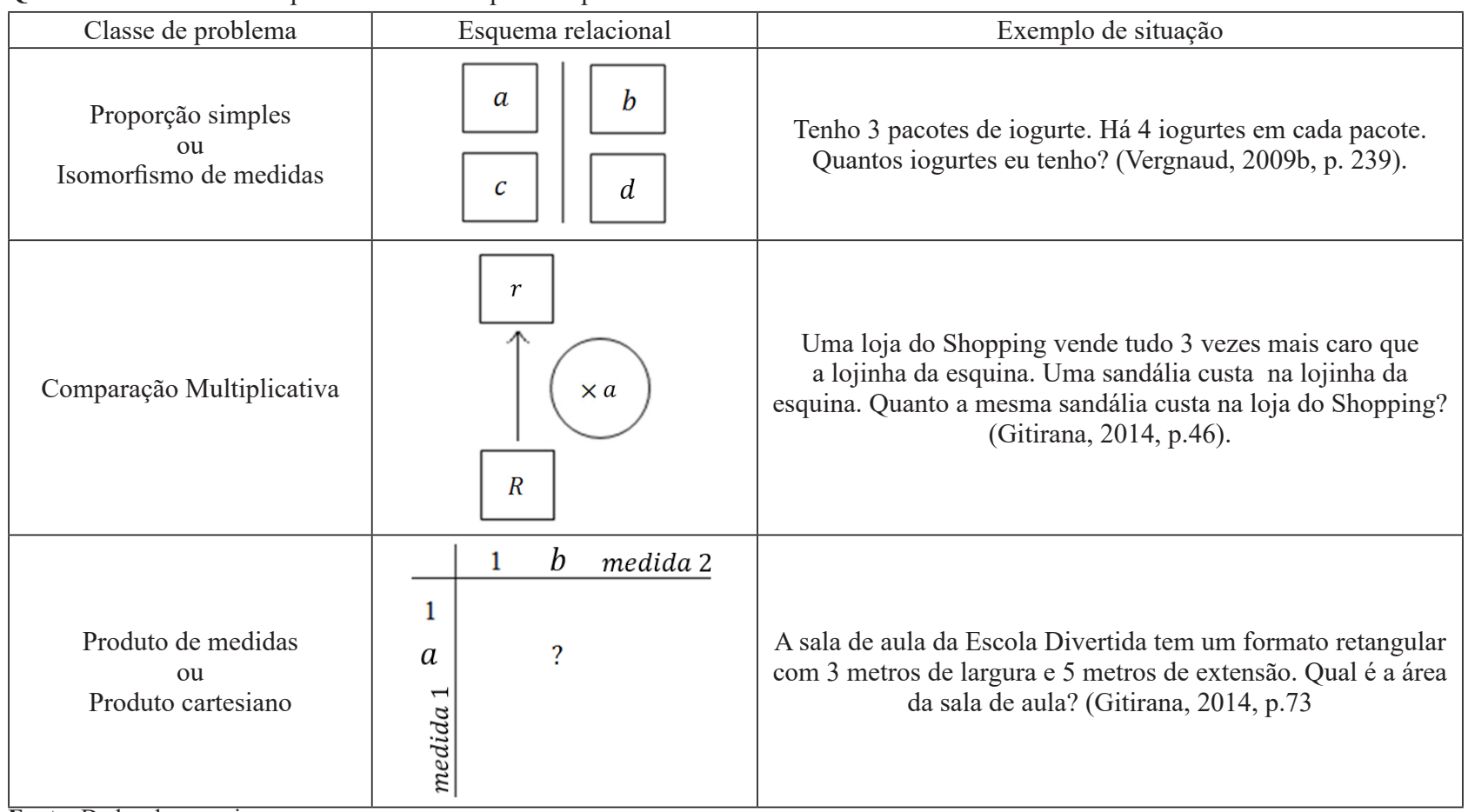

Fonte: Dados da pesquisa.

Para a segunda classe, comparação multiplicativa, caso a comparação multiplicativa seja vezes menor ou metade, a comparação é feita por meio de uma divisão, variação que pode ser um fator modificador da dificuldade dessa classe de situação. Na terceira classe, o produto de medidas pode ser feito entre medidas discretas, neste caso denominada por produto cartesiano, ou entre medidas contínuas.

A quarta classe de problemas das estruturas multiplicativas é denominada função bilinear ou proporção dupla. Segundo Gitirana (2014), esses tipos de problema são abordados no estudo de problemas de regra de três composta. Eles “[...] envolvem ao menos seis grandezas (três pares de mesma natureza), em que uma delas é proporcional a duas outras, separadamente" (Gitirana, 2014, p.81). Segundo Vergnaud (1993), esses problemas ligam as medidas duas a duas: por exemplo, proporcional a, proporcional a uma situação que ilustra essa categoria é: "Um parque de diversão cobra para cada criança brincar em qualquer brinquedo durante hora. Dona Lulu levou seus filhos para brincar no parque durante horas. Quanto ela pagou?" (Gitirana, 2014, p. 81). A situação pode ser apresentada pela Figura 3.
Figura 3 - Esquema relacional de um problema do tipo função bilinear

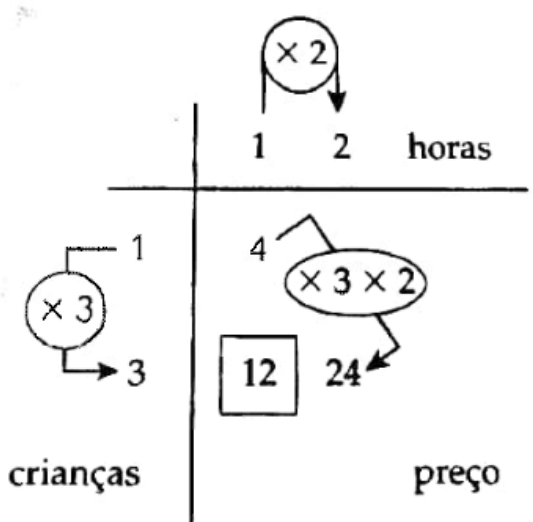

Fonte: Gitirana (2014, p. 84).

A quinta classe de problemas das estruturas multiplicativas é denominada proporcionalidade múltipla. Neste caso, uma medida é proporcional a e a, mas e são independentes entre si (Vergnaud, 1993).

Segundo Gitirana (2014), esta última classe de situações que dá significado à multiplicação trata-se de uma composição 
de duas proporções simples. Por exemplo: “A receita da massa de pastel do 'seu' Manoel é assim: para cada copo de leite ele usa 3 ovos, e para cada ovo, 2 xícaras de farinha. Para fazer a massa usando 2 copos de leite quantas xícaras de farinha ele vai precisar?" (Gitirana, 2014, p. 86).

Neste caso, a proposta de representação para a questão é o seguinte Figura 4:

Figura 4 - Representação do esquema relacional de uma problema do tipo proporcionalidade múltipla

\begin{tabular}{c|c|c} 
Copos de leite & Ovos & Copos de farinha \\
\hline 1 & 1 & 2 \\
2 & 3 & $?$
\end{tabular}

Fonte: Dados da pesquisa.

Vergnaud (2009b) menciona que os problemas de proporção múltipla permitem que sejam elaboradas perguntas intermediárias. Por exemplo, na situação anterior é possível, primeiro, questionar quantos ovos são utilizados na receita para dois copos de leite, para depois questionar quantas xícaras de farinha são necessárias para dois copos de leite.

Assim como as estruturas aditivas, as classes de problemas das estruturas multiplicativas também possuem subclasses de problemas, de acordo com as variáveis disponíveis e as questionadas na situação. Além disso, há outros fatores que interferem na diversidade e dificuldade das situações, referentes a: i) facilidade ou dificuldade maior do cálculo necessário (relacionada aos cálculos com números muito grandes, números inteiros, números decimais, entre outros); ii) apresentação das medidas envolvidas no problema (relacionada ao fato de mostrarem ou não valores que devem ou podem ser desconsiderados na situação, a ordem em que as informações aparecem, ordem temporal ou não); e iii) tipo do conteúdo e de relação.

A partir dos problemas do campo aditivo e do campo multiplicativo, Vergnaud (2009b) considera um terceiro tipo de problema, denominado problemas mistos, que se referem a situações que exigem operações de ambos os campos conceituais - aditivo e multiplicativo. Em outras palavras, esses problemas envolvem pelo menos uma adição e multiplicação simultaneamente (ou suas respectivas operações inversas, subtração e divisão).

Os problemas mistos são complexos, que apresentam várias relações e questões em jogo (Vergnaud, 2009b). De acordo com o pesquisador, o estudo das relações elementares apresentadas anteriormente é "[...] insuficiente para dar uma imagem completa das questões que existem na solução de problemas de aritmética" (Vergnaud, 2009b, p. 269).

No Quadro 3, apresentamos um problema misto proposto por Vergnaud (2009b), no qual nos baseamos para a análise dos problemas de função afim, foco da presente investigação.
Quadro 3 - Exemplo de problema misto

Um comerciante de camisas compra 3 dúzias de camisas a a dúzia e revende-as a a peça. Colocar as informações em uma tabela de correspondência fazendo a previsão de uma coluna para os lucros. Encontrar todas as perguntas que cabem nessa tabela e todos os caminhos que permitam encontrar apenas o lucro total do comerciante de camisas.

Fonte: Vergnaud (2009b, p. 288).

A representação sugerida por Vergnaud (2009b) para o referido problema misto permite observar as perguntas possíveis de ser propostas, de acordo com as medidas apresentadas no enunciado. Desta forma, temos as relações demonstradas na Figura 5.

Figura 5 - Esquema relacional de um problema misto

\begin{tabular}{|c|c|c|c|c|}
\hline $\begin{array}{l}\text { dúzias de } \\
\text { camisas }\end{array}$ & camisas & $\begin{array}{l}\text { preço de } \\
\text { compra }\end{array}$ & $\begin{array}{l}\text { preço de } \\
\text { venda }\end{array}$ & lucro \\
\hline & 1 & $B$ & $\rightarrow 40$ & $\mathrm{~F}$ \\
\hline $1-$ & $-12-$ & $\rightarrow 360$ & D & G \\
\hline 3 & A & C & $\mathrm{E}$ & $\mathrm{H}$ \\
\hline
\end{tabular}

Fonte: Vergnaud (2009b, p.289).

Segundo Vergnaud (2009b), as perguntas relacionadas a cada letra em caixa alta na Figura 2 são:

A Número total de camisas;

B Preço de compra de uma camisa;

C Preço de compra de três dúzias de camisas;

D Preço de venda de 12 camisas;

E Preço de venda de 3 dúzias de camisas;

F Lucro em 1 camisa;

G Lucro em 12 camisas; e

$\mathrm{H}$ Lucro em 3 dúzias de camisas.

Vergnaud (2009b) sugere que o modo de representação das medidas seja um dos princípios indispensáveis para a análise profunda das relações e transformações em jogo nesses problemas, pois a resolução deles acontece pelo desenvolvimento da prática de formular perguntas intermediárias, sem que estas tenham relação direta com o enunciado.

Uma vez considerada a representação proposta na figura 2, de acordo com Vegnaud (2009b), é fácil identificar possíveis caminhos para determinar a solução da situação, como por exemplo: BFGH: cálculo do preço da compra (B) cálculo do lucro de 1 camisa (F) cálculo do lucro para 12 camisas (G) cálculo do lucro para três dúzias $(\mathrm{H})$.

Neste contexto, estabelecemos como hipótese que os problemas de função afim são passíveis de ser associados aos problemas mistos, conforme caracterizados por Vergnaud (2009b), e que a partir das classificações das estruturas aditivas e multiplicativas, é possível estabelecer uma classificação aos problemas de função afim.

Os procedimentos metodológicos e as análises da pesquisa estão apresentados a seguir. 


\section{Procedimentos Metodológicos e Análise dos Dados}

A presente pesquisa caracteriza-se como qualitativa. As análises dos problemas de função afim são realizadas a partir das classes de situações estabelecidas por Vergnaud para os campos conceituais aditivo e multiplicativo. O livro didático de Matemática caracterizou-se como uma fonte para a coleta de informações, pois a partir da obra identificamos situaçõesproblema relacionadas ao conceito de função afim.

A coleção de livros didáticos adotada para esta pesquisa é a Matemática: interação e tecnologia (Balestrini, 2016), aprovada pelo Programa Nacional do Livro Didático PNLD 2018 e selecionada para esta investigação por ser a mais adotada pelas escolas públicas do Núcleo Regional de Educação de Campo Mourão - Paraná. O volume 1 foi considerado para as análises por conter um capítulo dedicado ao estudo da função afim.

Para este artigo, apresentamos as análises de cinco situações-problema do capítulo específico do livro didático que trata de função afim, sendo os problemas propostos no início do capítulo do livro como motivação para a introdução ao estudo do conceito de função afim, e exemplos resolvidos. As situações-problema analisadas se restringem àquelas que envolvem um contexto externo à matemática.

De acordo com Vergnaud (2009b), essas situações possuem um conteúdo e um tipo de relação a ser compreendida entre as medidas apresentadas como, por exemplo, quantidade de bolinhas de gude, valores em dinheiro, quilômetros percorridos, entre outros, e a relação pode ser, por exemplo, uma relação ternária de transformação - ganhar ou perder.

Com base na proposta de Vergnaud (2009b) para a análise de problemas mistos, organizamos os dados das situações em quadros, nos quais as colunas se referem a medidas de mesma natureza, representando por setas as relações estabelecidas entre medidas; e por letras, medidas desconhecidas, que no caso das funções, as variáveis assumem a posição de uma medida de um conjunto relacionado a uma determinada grandeza.

As representações dos esquemas relacionais foram elaboradas com base nos códigos estabelecidos por Vergnaud, auxiliando na visualização do tipo de relação estabelecida entre as medidas. Por exemplo, utilizamos flechas para indicar a relação de transformação ou comparação entre dois elementos da situação, e chaves para indicar composição de medidas.

As relações estabelecidas entre as medidas presentes no enunciado de cada situação-problema proposta foram identificadas e relacionadas a uma das cinco estruturas de problemas do campo conceitual multiplicativo e/ou uma das seis estruturas de problemas do campo conceitual aditivo. Por fim, classificamos os problemas em mistos ou não, de acordo com as estruturas identificadas nas relações entre medidas. Análise do problema 1:

Na obra (Balestrini, 2016), o conceito de função afim é introduzido a partir do contexto de uma corrida de táxi. Esta situação permite a representação algébrica de uma função afim quando estabelecida a relação entre os quilômetros percorridos e o valor em dinheiro a ser pago ao final da corrida, conforme Figura 3.

Figura 6 - Situação introdutória de função afim

\section{- DEFINIÇÃO DE FUNÇÃO AFIM}

Geralmente o valor cobrado pelas carridas de táxi é composto de uma parte fixa, conhecida como bandeirada, e de outra parte cor respondente à quantidade de quilômetros percorridos. supondo que em uma cidade a bandeirada custe $R \$ 5,00$ e o quilömetro percorrido R\$ 2,00, é possivel escrever uma fórmula que permita calcular o valor a ser paga por uma corrida em função da quantidade de quilômetros percorridos. Para escrever essa fórmula, utilizaremos y (variável dependente) para representar a valor, em reais a ser pago pela corrida e $x$ (variável independente) para representar a quantidade de quilômetros percorridos.

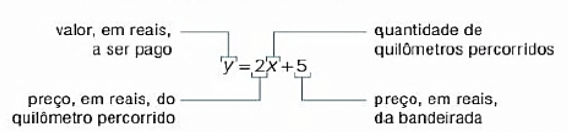

Fonte: Balestri (2016, p. 68).

Com base em Vergnaud (2009, p. 289), para a análise de um problema misto, construímos o esquema a seguir (Figura 7), que permite uma análise detalhada das informações e perguntas pertinentes à situação e das relações estabelecidas entre as medidas presentes na situação-problema.

Figura 7 - Esquema relacional para a análise do problema 1

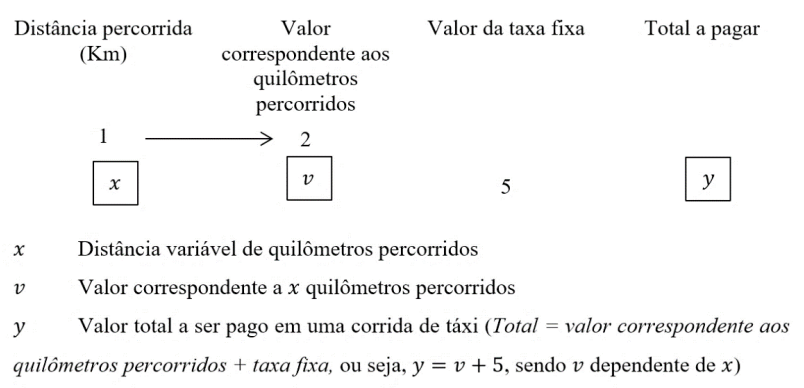

Fonte: Dados da pesquisa.

Neste esquema, optou-se por utilizar a letra para referir a distância de quilômetros percorridos, e ao total a ser pago ao fim da corrida, para que a expressão algébrica da situação seja proposta em termos de e , como usualmente adotada em livros didáticos. Neste caso, tem-se representado em função de , permitindo substituir y por .

Analisando o esquema de resolução para o problema 1 , identificamos que uma das perguntas a ser feita é "qual o total a ser pago ao percorrer ?". Esta pergunta pode ser resolvida realizando uma multiplicação () e uma adição (Total = valor correspondente aos quilometros percorridos + taxa fixa). Esta consideração não é suficiente quando o objetivo é representar a relação para percorridos em uma corrida de táxi, mas permite analisar o cálculo necessário para que se possa responder à referida pergunta, dando respaldo para determinar posteriormente o valor da corrida para uma quantidade qualquer de quilômetros rodados.

Ainda considerando o esquema proposto para a resolução do problema 1, nas duas primeiras colunas consideramos a existência de uma relação quaternária, uma correspondência entre elementos (medidas) de conjuntos de natureza distinta, 
sendo as medidas envolvidas diretamente proporcionais. Assim, podemos representar a relação:

Distância percorrida $(\mathrm{Km})$

Valor correspondente $(\mathrm{R} \$)$

1

$x$

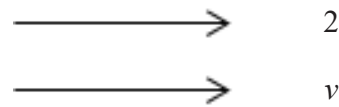

Logo, uma interpretação possível é que está para , assim como está para, que algebricamente é representado por:

$$
\frac{1}{x}=\frac{2}{v}
$$

Trata-se da estrutura proporção simples, de uma situação do campo conceitual multiplicativo, cuja representação algébrica permite uma análise dimensional²:

$$
\frac{1 \mathrm{~km}}{x \mathrm{~km}}=\frac{2 \text { reais }}{v \text { reais }}
$$

Para determinar o total a ser pago por uma corrida de táxi, é preciso, ainda, determinar a primeira parcela de uma soma, o valor a ser pago que corresponde aos quilômetros percorridos (). Assim, para determinar, basta multiplicar ambos os lados da igualdade por reais e por ().

$$
\begin{gathered}
\text { vreais }=2 \text { reais } \times \frac{x \mathrm{~km}}{1 \mathrm{~km}} \\
\text { vreais }=\frac{2 \text { reais } \times x \mathrm{~km}}{1 \mathrm{~km}} \\
\text { vreais }=2 x \text { reais }
\end{gathered}
$$

A segunda relação que devemos considerar é aquela cujo resultado representa o total a ser pago, que se trata da composição de duas medidas de mesma natureza (reais), total a pagar = valor correspondente aos quilometros percorridos + taxa fixa. Interpretamos que se trata de um problema de composição de medidas do campo aditivo, que pode ser representado pelo seguinte esquema relacional (Figura 8):

Figura 8 - Esquema relacional da composição de medidas identificada no Problema 1

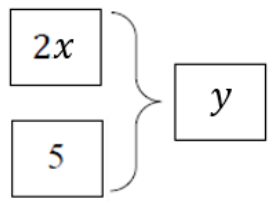

Fonte: Dados da pesquisa.

Este esquema pode ser expresso algebricamente por:

$$
\mathrm{y}=2 \mathrm{x}+5 \text {. }
$$

Assim, a situação-problema analisada se trata de um problema misto, pois há uma relação multiplicativa do tipo proporção simples, quando obtemos o valor a ser pago de acordo com os quilômetros percorridos; e uma relação aditiva do tipo composição de medidas, quando definimos o total a ser pago composto pelo valor que depende dos quilômetros percorridos mais a taxa fixa, sendo estes valores em dinheiro a serem juntados.

Interpretando estas informações na forma de uma relação funcional, temos $\mathrm{y}=2 \mathrm{x}+5$ ou $\mathrm{f}(\mathrm{x})=2 \mathrm{x}+5$, representando a função do total a ser pago e a relação de dependência entre o total pago e a distância percorrida em .

Análise do problema 2:

O segundo exemplo analisado está dividido em duas partes, e seu objetivo é o estudo de funções crescente e decrescente, conforme apresentado na figura 9.

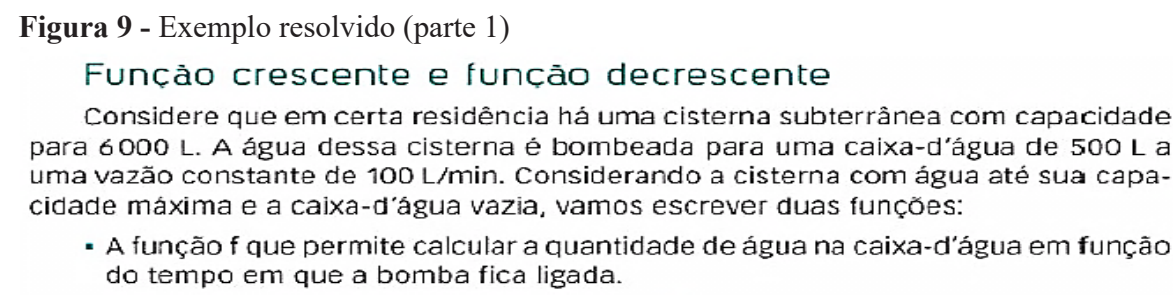

Para esta primeira parte do problema, a função solicitada relaciona a quantidade de água acumulada na caixa d'água em um tempo . Esta é uma relação quaternária, pois coloca em correspondência dois conjuntos distintos em uma relação de proporção direta. A correspondência entre os conjuntos pode ser verificada pela chamada constante de vazão de, ou seja, pode-se estabelecer a correspondência que em 1 minuto haverá 100 litros de água.

Para determinar a função que expressa a quantidade de água na caixa d'água em minutos, consideremos a relação quaternária, proporção simples, como representado a seguir:

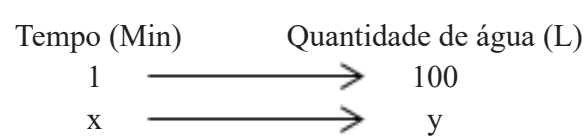

Desse modo, podemos afirmar que 1 está para assim como está para, e escrevermos:

$$
\begin{gathered}
\frac{1 \text { minuto }}{x \text { minutos }}=\frac{100 \text { litros }}{y \text { litros }} \\
y \text { litros }=\frac{100 \text { litros } \times x \text { minutos }}{1 \text { minuto }} \\
y \text { litros }=100 x \text { litros }
\end{gathered}
$$


Assim, a função correspondente à quantidade de água que entra na caixa d'água é ou .

Podemos interpretar a quantidade de água que entra na caixa d'água como a quantidade de água que sai da cisterna, pois há uma equivalência entre as relações sair água da cisterna e entrar água na caixa, considerando a direção do fluxo de água (Figura 10).

Figura 10 - Interpretação do problema 2 (parte 1)



Fonte: Dados da pesquisa.

Esta interpretação é importante para a segunda parte do problema da cisterna, apresentado na Figura 11. Na segunda parte desta situação, notamos que a quantidade de água da cisterna passa por uma transformação negativa, pois a água da cisterna está sendo retirada.

Figura 11 - Exemplo resolvido (parte 2)

E a função g que permite calcular a quantidade de água na cisterna em função do tempo em que a bomba fica ligada. litros

Fonte: Balestri (2016, p.75).

Neste caso, podemos colocar a relação na forma estadoinicial, transformação, estado-final, sendo o estado-inicial a quantidade inicial de água na cisterna e o estado-final a quantidade de água restante na cisterna. Já a transformação refere-se à quantidade de água que sai da cisterna (e esta última já foi calculada na primeira parte do problema, sendo necessário considerar a relação como equivalente à água que entra na caixa).

Neste caso, identificamos que a transformação é um número relativo negativo, pois a relação estabelecida é a água sair da cisterna. Assim, esta situação-problema constitui, primeiramente, um problema multiplicativo, para determinação da quantidade de água que sai da cisterna (ou que entra na caixa d'água) - verificada na primeira parte da situação (Figura 9) - e, em um segundo momento, um problema aditivo, do tipo transformação de medidas. Considerando que o estado inicial da cisterna é sua capacidade total (), indicamos a relação pelo esquema relacional que segue:

Figura 12 - Esquema relacional da transformação de medidas identificada no problema 2 (parte 2)

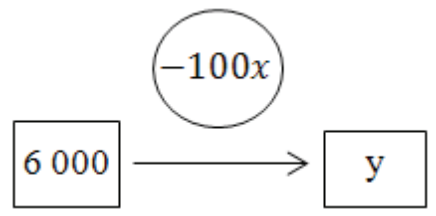

Fonte: Dados da pesquisa.
Desta relação, escrevemos a equação $y=6000-100 x$, ou como solicitado pela situação, $\mathrm{g}(\mathrm{x})=-100 \mathrm{x}+6000$.

Identificamos esta situação-problema como um problema misto, pois constatamos uma relação aditiva em que há um estado-inicial, um estado-final e uma transformação negativa, uma situação de transformação de medidas, sendo a transformação o resultado de uma relação multiplicativa que está relacionada à taxa de vazão da água, tratando-se de um problema multiplicativo de proporção simples.

Análise do problema 3:

A terceira situação analisada refere-se a um reservatório de água, e está apresentada na Figura 13.

Figura 13 - Exemplo resolvido - Reservatório

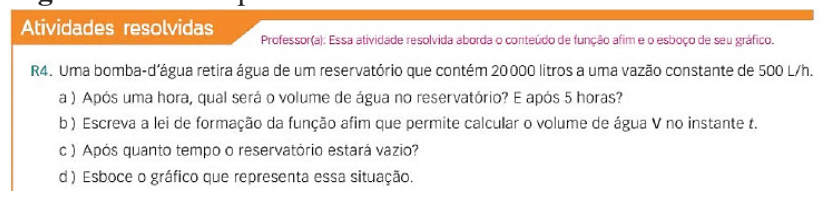

Fonte: Balestri (2016, p.78).

A primeira pergunta diz respeito a um problema com medidas numéricas do campo multiplicativo, pois relaciona quatro medidas, colocadas duas a duas em correspondência, sendo essas diretamente proporcionais.

O segundo item solicita a escrita de uma relação no instante minutos. Este se assemelha ao problema resolvido anteriormente, inclusive pelo contexto (água retirada de um lugar para outro).

No que se refere à taxa de vazão da água para fora do reservatório, é preciso considerar a relação de proporção simples para obter a quantidade de água que é retirada do reservatório em horas:

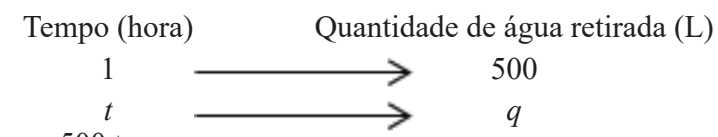

ou seja, q=500 t.

No que se refere ao volume () de água restante no reservatório após horas, é preciso considerar o estado-inicial, reservatório totalmente cheio; e o estado-final, a quantidade de água após t horas, tratando-se de uma relação de transformação de medidas de mesma natureza, pertencente ao campo aditivo. Dada a quantidade de água que sai do reservatório por horas, o volume de água após horas pode ser representado pelo seguinte esquema relacional (Figura 14):

Figura 14 - Esquema relacional da transformação de medidas identificada no problema 3

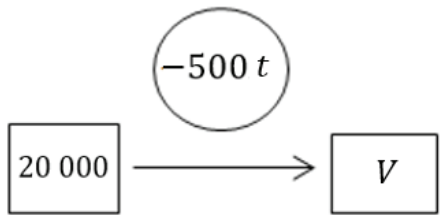

Fonte: Dados da pesquisa.

Assim, V=20 000-500·t.

Como no problema 2, no problema 3 questiona-se o estado-final de uma transformação negativa, pois se interroga 
a quantidade de água ainda reservada após a saída de uma determinada quantidade de água. Portanto, estas duas situações (Figuras 5 e 6) pertencem a uma mesma classe de problemas mistos.

Ressaltamos que, para Vergnaud, é importante a diversidade de situações para a aprendizagem do estudante. No entanto, o autor da obra analisada optou por apresentar duas situações semelhantes, do ponto de vista da teoria dos Campos Conceituais, uma seguida da outra, fato que pouco contribui para ampliar os esquemas de resolução desses problemas pelos estudantes.

Análise do problema 4:

A quarta situação-problema analisada (Figura 15) trata-se de um problema introdutório do caso específico de função linear. Este é um problema puramente multiplicativo, sinalizado por Vergnaud (2009b) por ter relação com os conceitos de função linear e proporcionalidade, e por não envolver a parte aditiva (coeficiente linear) da função afim. O problema aborda uma relação quaternária e, portanto, da relação entre quatro medidas, duas a duas de mesma natureza em uma relação de proporção direta.

Figura 15 - Problema introdutório de função linear

Exemplo: Professor(a): Caso seja necessário, diga aos alunos que duas grandezas inversamente proporcion O rendimento médio de soja (em grão) no Brasil na safra de 2014/2015 foi de aproximadamente 3 toneladas por hectare. Vamos representar essa situação com a função linear:

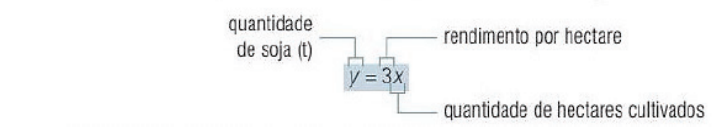

Fonte: Balestri (2016, p.77).

A expressão 3 toneladas por hectare coloca em relação os conjuntos toneladas e hectares. Para obter a função que representa a quantidade de soja aproximada () medida em toneladas, em função da quantidade de hectares cultivados (), fazemos a correspondência entre e . Entende-se que está para assim como está para, como representado no esquema seguinte:

Logo,

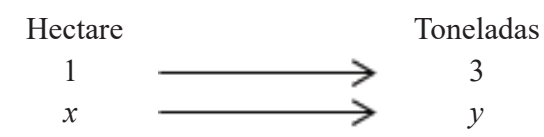

$$
\begin{aligned}
& \frac{1 \text { hectare }}{x \text { hectares }}=\frac{3 \text { toneladas }}{y \text { toneladas }} \\
& y \text { toneladas }=\frac{3 \text { toneladas } \times x \text { hectare }}{1 \text { hectares }} \\
& y \text { toneladas }=3 x \text { toneladas } \\
& y=3 x
\end{aligned}
$$

Análise do problema 5:

O quinto problema analisado também é relacionado à função linear, ou seja, caso particular de função afim, quando $\mathrm{b}=0$. A situação-problema proposta é: "Marcela abasteceu seu veículo com 151 de etanol, e pagou $R \$ 42,75$ por esse combustivel. Quanto ela pagaria se tivesse abastecido com 401 de etanol?" (Balestri, 2016, p. 80). Esta situação é proposta, inicialmente, como um problema pertencente ao campo multiplicativo, pois há uma relação quaternária, duas a duas de mesma natureza, com uma medida desconhecida.

Neste caso, as medidas em que as relações se estabelecem são litros de etanol e valor pago. De acordo com o contexto proposto, temos que 15 litros de etanol estão para 40 litros de etanol, assim como 42,75 reais estão para reais.

$$
\frac{15}{40}=\frac{42,75}{q}
$$

Segundo Vergnaud (2009b), este tipo de problema é considerado complexo, pois " [...] nenhuma das quatro quantidades é a unidade e [...] a regra de três a que se chega, nesse caso, é uma regra de três não deturpada (denominador diferente de 1)" (p. 246). Em outras palavras, após isolar a incógnita haverá, como resultado, uma multiplicação e uma divisão, cujo denominador é diferente de 1 , como verificado neste caso:

$$
\begin{gathered}
15 \cdot q=40 \cdot 42,75 \\
q=\frac{40 \cdot 42,75}{15}
\end{gathered}
$$

Em seguida, o autor do livro didático propõe como sequência do problema a situação apresentada na Figura 16.

Figura 16 - Exemplo resolvido - Função linear De maneira semelhante, vamos obter a lei de formaçāo da funçẫo cue representa essa situaçāo. Para isso, calculamos quanto Marcela pagaria por $x$ litros de etanol.

\begin{tabular}{|c|c|}
\hline Etanol (L) & Valor (R\$) \\
\hline 15 & 42,75 \\
\hline$x$ & $y$ \\
\hline
\end{tabular}

$$
\begin{aligned}
\frac{15}{x} & =\frac{42,75}{y} \\
15 \cdot y & =x \cdot 42,75 \\
y & =\frac{42,75}{15} \cdot x \\
y & =2,85 x
\end{aligned}
$$

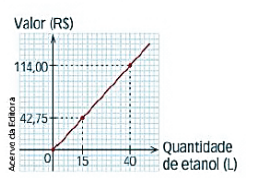

Fonte: Balestri (2016, p.80).

Este problema pode ser resolvido de forma análoga ao problema introdutório à função linear (Figura 15), pois se trata de um problema de proporção simples, conforme esquema a seguir.



Este esquema pode ser representado algebricamente por:

$$
\frac{15}{x}=\frac{42,75}{y}
$$

Segundo Vergnaud (2009b), uma forma de analisar situações-problema do tipo multiplicativo em que não há correspondência com a unidade e que resulta uma regra de três não deturpada é realizar uma análise vertical do esquema proposto. Este tipo de análise permite passar de uma linha para outra, e se trata de multiplicar ou dividir por um operadorescalar (sem dimensão) para obter a correspondência com a unidade.

A pergunta pertinente para esta análise é: "Qual o valor a ser pago por 1 litro de Etanol?" e a representação para este cálculo relacional pode ser dada por: 


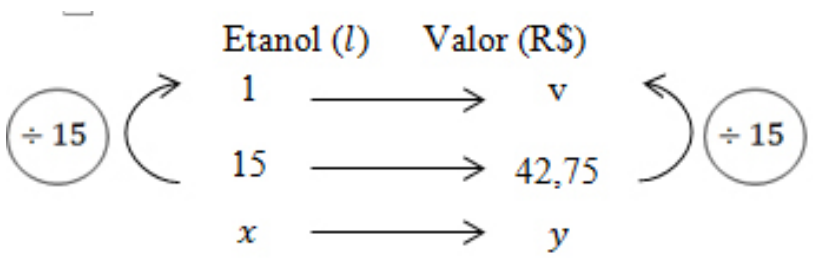

Sendo assim:

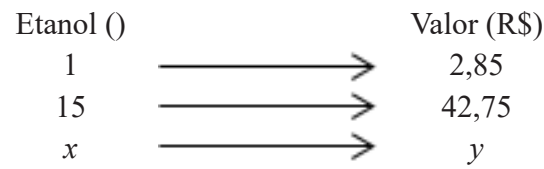

Considerando a representação acima e a proporcionalidade envolvida, pode-se escrever:

$$
\frac{1}{2,85}=\frac{15}{42,75}=\frac{x}{y}
$$

ou seja,

$$
\frac{1}{2,85}=\frac{x}{y}
$$

Esta situação-problema é considerada mais complexa para os alunos por não apresentar a equivalência com a unidade, e por envolver números decimais.

As análises das situações-problema foram realizadas com base na análise do problema misto proposto por Vergnaud (2009b). Para isso, organizaram-se as quantidades por meio de esquemas, com a intenção de identificar as medidas e as relações estabelecidas entre elas. A partir dessa organização e identificação, foi possível identificar as estruturas dos problemas analisados com a(s) relação(ões) estabelecida(s) e que coincide(em) com as categorias de situações relacionadas às Estruturas Aditivas e às Estruturas Multiplicativas.

Vergnaud (2009b) menciona que não apresentou uma classificação para os problemas mistos devido à diversidade de situações e relações a serem consideradas. Contudo, os resultados desta investigação mostram essa possibilidade, confirmando a nossa hipótese de pesquisa, de que os problemas de função afim são passíveis de ser associados aos problemas mistos e que, a partir das classificações das estruturas aditivas e multiplicativas, é possível estabelecer uma classificação para os problemas envolvendo função afim. Dito de outra forma, é legítimo considerar a possibilidade de estabelecer uma classificação para as situações que dão sentido ao conceito de função afim.

\section{Considerações Finais}

Os resultados desta pesquisa mostram que as situaçõesproblema relacionadas ao conceito de função afim são passíveis de ser associadas a problemas mistos, envolvendo operações do campo aditivo e do campo multiplicativo ou a problemas estritamente multiplicativos.

As análises revelam que as situações-problema que se referem a uma função linear, ou seja, os casos em que o coeficiente linear é igual a, como o quarto e o quinto problemas analisados, são puramente multiplicativas, pertencentes estritamente ao campo conceitual multiplicativo, e são classificadas como situações do tipo proporção simples (Vergnaud, 2009a; 2009b). Apesar de os problemas 4 e 5 pertencerem a uma mesma classe de problemas - proporção simples -, o quinto problema analisado possui variação quanto à sua estrutura, que indica um nível de dificuldade mais elevado que o problema 4. Isto ocorre devido a dois fatores: o resultado de uma regra de três não deturpada, ou seja, com denominador diferente de 1 (Vergnaud, 2009b); e cálculos envolvendo números decimais.

Os três primeiros problemas analisados são classificados como problemas mistos, pois envolvem operações aditiva e multiplicativa, sendo o problema 1 composto por uma proporção simples (campo multiplicativo) e uma composição de medida (campo aditivo); e os problemas 2 e 3, compostos por uma proporção simples (campo multiplicativo) e uma transformação de medidas (campo aditivo).

Por meio deste artigo, apresentamos o início de nossos estudos, que visam a mapear o campo conceitual das funções, e estabelecer uma classificação para as situações-problema de função afim. O caminho que o GEPeDiMa traçou para o mapeamento do campo conceitual da função afim provavelmente será longo, e os resultados desta investigação constituem um primeiro passo para o estabelecimento de uma classificação das situações que dão significado a este conceito. Assim, foi possível mostrar que as situações de função afim podem ser associadas a problemas mistos ou a problemas do tipo multiplicativo puro, e que é possível, em ambos os casos, estabelecer uma classificação para esses problemas. Estabelecer a tipologia das situações é seguramente o passo mais importante, uma vez que elas constituem a porta de entrada do campo conceitual que intentamos mapear no âmbito do GEPeDiMa.

Para além das contribuições teóricas, os estudos aqui apresentados trazem respaldo para as ações dos professores de Matemática em sala de aula, pois a partir dessas classificações é possível ter ciência e selecionar diferentes situações com variação nas estruturas dos enunciados, possibilitando a elaboração de novos esquemas pelos estudantes. Neste sentido, ao serem progressivamente desenvolvidas em sala de aula, a variação dessas situações, no decorrer do processo escolar, proporciona aos alunos a aprendizagem do conceito de função afim.

\section{Referências}

Balestri, R. (2016). Matemática: Interação e Tecnologia. São Paulo: Leya.

Brasil. (2019). Base Nacional Comum Curricular. Brasília: MEC.

Campiteli, H. C. \& Campiteli, V. C. (2006). Funções. Ponta Grossa: UEPG.

Gitirana, V. (2014). Repensando multiplicação e divisão: contribuições da teoria dos campos conceituais. São Paulo: PROEM. 
Vergnaud, G. (1993). Teoria dos Campos Conceituais. In: Anais do $1^{\circ}$ Seminário Internacional de Educação do Rio de Janeiro. IM UFRJ. pp.1-26.

Vergnaud, G. (2009a). O que é aprender. In: M. Bittar, Marilena, C.A, Muniz. A aprendizagem matemática na perspectiva da Teoria dos Campos Conceituais, (pp.13-35). Curitiba: CRV.

Vergnaud, G. (2009b). A criança, a matemática e a realidade: problemas do ensino da matemática na escola elementar. Curitiba: UFPR.

Vergnaud, G. (2011). O longo e o curto prazo na aprendizagem da matemática. Educar em Revista, (esp.), 15-27.

Zanella, M.S. \& Barros, R. M. O. (2014). Teoria dos Campos Conceituais: situações problemas da estrutura aditiva $e$ multiplicativa de naturais. Curitiba: CRV. 